\title{
Microsymposium
}

MS063.002

\section{Regulation of RING ubiquitin ligases by small protein molecules}

\author{
Danny Huang ${ }^{1}$ \\ ${ }^{1}$ Cancer Research UK Beatson Institute, Glasgow, United Kingdom \\ E-mail: d.huang@beatson.gla.ac.uk
}

Post-translational modification by ubiquitin (Ub) regulates diverse cellular processes. Ubiquitin ligases (E3s) catalyze the final step of Ub transfer from E2 ubiquitin-conjugating enzyme thioesterified with Ub (E2 Ub) to a lysine side chain of substrate. RING-type E3s are the largest family of E3s with approximately 600 members in humans. RING E3s function by recruiting E2 Ub via the RING domain and promote direct transfer of Ub from E2 to the substrate lysine. The extent of substrate ubiquitination depends on the processivity of the RING E3-E2 Ub complex. RING domain activates E2 Ub by stabilizing E2 Ub in the closed conformation such that the thioester bond is optimally oriented for nucleophilic attack by the substrate lysine. Moreover, we showed that non-covalent Ub binding to the "backside" of E2 UbcH5B stimulates the catalytic efficiency of the RING E3-E2 Ub complex by enhancing RING E3's affinity for E2 Ub complex. Recently we have developed small protein molecules that modulate the activity of RING E3s. I will present these results in this meeting

Keywords: Ubiquitin, RING E3, ligase 